\title{
YAWNING ANALYSIS FOR DRIVER DROWSINESS DETECTION
}

\author{
G. M. Bhandari ${ }^{1}$, Archana Durge ${ }^{2}$, Aparna Bidwai ${ }^{3}$, Urmila Aware ${ }^{4}$ \\ ${ }^{1,2,3,4}$ Computer Department, JSPM's Bhivarabai Sawant Institute Of Technology and Research(W), Maharashtra, India
}

\begin{abstract}
Driver fatigue is the main reason for fatal road accidents around the world. In this paper, an efficient driver's drowsiness detection system is designed using yawning detection.Here, we consider eye detection and mouth detection. So that road accidents can avoid successfully. Mouth features points are identified using the redness property. Firstly detecting the driver's face using YCbCr method then face tracking will perform using canny edge detector. After that, eyes and mouth positions by using Haar features. Lastly yawning detection is perform by using mouth geometric features. This method is tested on images from videos. Also proposed system should then alert to the driver in case of inattention.
\end{abstract}

Keywords: Face detection, Face tracking, Eye and Mouth detection, Yawn detection - ***

\section{INTRODUCTION}

Drowsy driver detection system is one of the potential applications of intelligent vehicle system. Driver fatigue is one of the main reasons causing traffic accidents. According to the National Highway Traffic Safety Administration (NHTSA) estimates, 100000 police reported crashes are directly cause by driver fatigue each year, which results in an estimated 1550 deaths, 71000 injuries, and $\$ 12.5$ billion losses [1]. In 2002, the National Sleep Foundation (NSF) reported that $51 \%$ adult drivers had driven a vehicle while feeling drowsy and $17 \%$ had actually fallen sleep.

If driver get a caution one second earlier than accident we can prevent accidences up to $90 \%$ so, for accident prevention, development technologies for monitoring the driver fatigue is require. Yawning is the important evidence of fatigue. In yawning state, mouth open wide and the geometric features of the mouth change obviously. This system should then alert the driver in the case of sleepiness or inattention. This paper contains the detail description about face detection, face tracking, eye and mouth detection, and yawning detection in system design part.

\section{SYSTEM DESIGN}

The driver drowsiness monitoring using yawning detection consists of different modules to analyze changes in the mouth of the driver. These modules are as follows:

1. Face detection

2. Face tracking

3. Eye and mouth detection

4. Yawning detection
The flow of our system as follows:

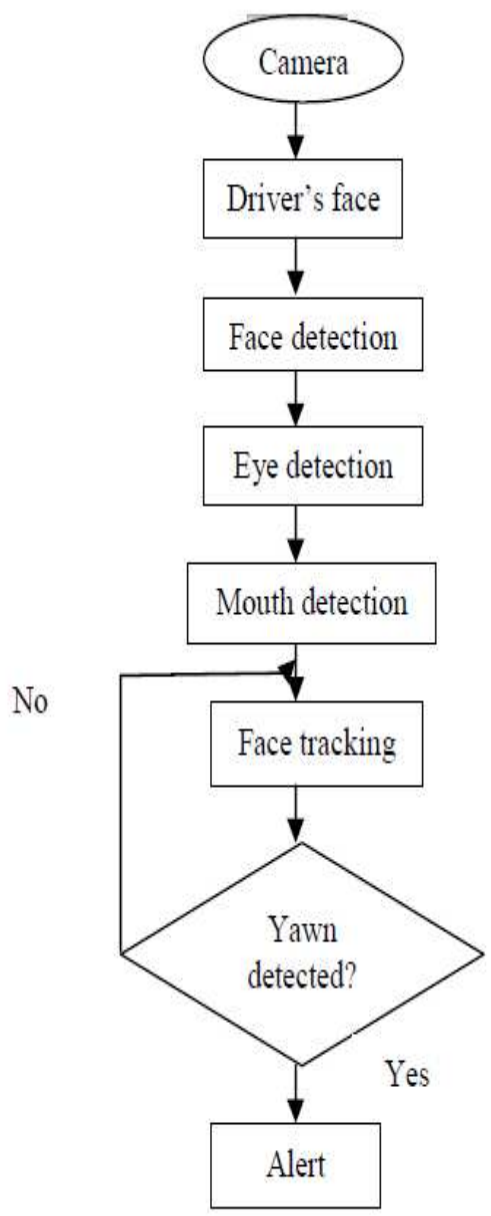

Fig 1: Flow Diagram 


\subsection{Face Detection}

It is the first step towards the system design. The most functional way to detect face is by detecting the skin color and texture. For skin detection, various color spaces like RGB and $\mathrm{YCbCr}$ are used and transformations in these spaces are performing. Skin detection is the process of finding skin colored pixels and regions in an image or a video. Skin detection process is referring as preprocessing step to find regions that potentially have human faces. The function of skin detectors is to transform a given pixel into an appropriate color spaces and then use a skin classifier to label the pixel whether it is a skin or non-skin pixel.

As shown in fig-2 given an input image to RGB color space and then transferred into $\mathrm{YCbCr}$. The result of this process is in black and white image representing face to white and background area around driver to black color.

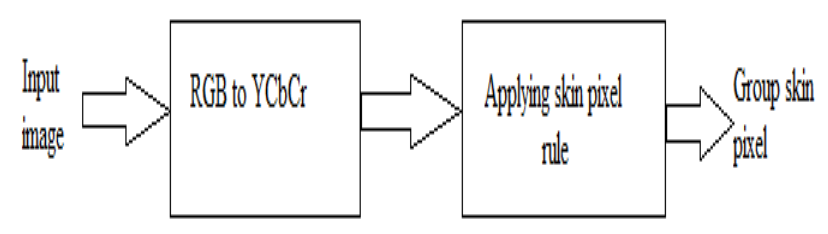

Fig 2: Skin detection

\subsubsection{RGB Color Space}

It is the most commonly used color space in digital images. It encodes colors as an additive combination of three primary colors: red (R), green (G) and blue (B). These colors are added together to form broad array of colors.

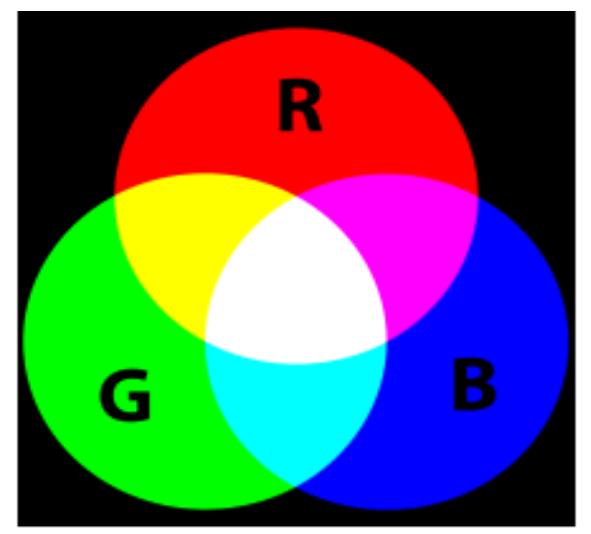

Fig 3: RGB color Model

As shown in figure 3 adding red to green yields yellow, adding all three primary colors together yields white, adding red to blue yields magenta, adding green and blue yield cyan.

\subsubsection{YCbCr Color Model}

$\mathrm{YCbCr}$ is a family of color spaces used as a part of the color image pipeline in video and digital photography systems. $\mathrm{Y}$ is a luma component and $\mathrm{Cb}$ and $\mathrm{Cr}$ are the blue difference and red difference chroma components. The difference between blue component and reference value component is called as $\mathrm{Cb}$ while the difference between the red component and reference value is referred to as component $\mathrm{Cr}$. $\mathrm{Y}$ is constructed as a weighted sum of RGB component and the $\mathrm{Cb}$ and $\mathrm{Cr}$ components are obtained by subtracting $\mathrm{Y}$ from respectively blue and red RGB components as [3]:

$$
\begin{gathered}
\mathrm{Y}=0.299 \mathrm{R}+0.587 \mathrm{G}+0.11 \mathrm{~B} \\
\mathrm{Cb}=\mathrm{B}-\mathrm{Y} \\
\mathrm{Cr}=\mathrm{R}-\mathrm{Y}
\end{gathered}
$$

YCbCr model of an acquired image as two set of static threshold ranges of $77<=\mathrm{Cb}<=127$ and $133<=\mathrm{Cr}<=173$ were proposed as skin pixel band.

\subsection{Face Tracking}

In face tracking, we can use the detected face as a template in next phases. Face tracking is done using canny edge detector. Canny egde detection:

It is an edge detection operator that uses a multistage algorithm to detect a wide range of edges in images. Canny aim was to discover the optimal edge detection algorithm in this situation an optimal edge detector means

1. Good detection: The algo should mark as many real edges in the images as possible.

2. Good localization: Edge mark should be as close as possible to the edges in the real images.

3. Minimal response: A given edge in the image should only be marked once and where possible image noise should not create false edge

\subsection{Eye and Mouth Detection:}

Eye and mouth detection is done using Haar like features. Voila and Jones devised an algorithm called Haar classifiers to rapidly detect any object including human faces using Adaboost classifier cascade that are based on Haar like features and integral image [2].

Integral images can be defined as two dimensional look up tables in the form of a matrix with the same size of the original image. Each element of the integral image contain the sum of all pixels located on the up-left region of the original image. This allow to compute sum of rectangular areas in the image at any position or scale, using only four look up's. 


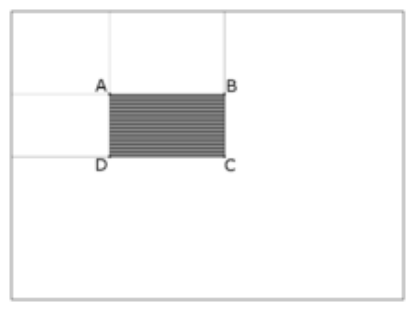

Fig 4: Integral Image

Finding sum of shaded rectangular area,

$$
\text { Sum }=\mathrm{I}(\mathrm{C})+\mathrm{I}(\mathrm{A})-\mathrm{I}(\mathrm{B})-\mathrm{I}(\mathrm{D})
$$

Haar like features are used in object recognisation. A Haar like features consider adjacent rectangular regions at a specific location in a detection window sum up the pixel intensities in each region and calculates the difference between these sums. A simple rectangular Haar like features can be defined as the difference of the sum of the pixels of area inside the rectangle which can be at a position and scaled within the original image. This features set is called 2-rectangular features. As shown in below figure 5 different Haar features as 2rectangular, 3-rectangular and 4-ractangular features.
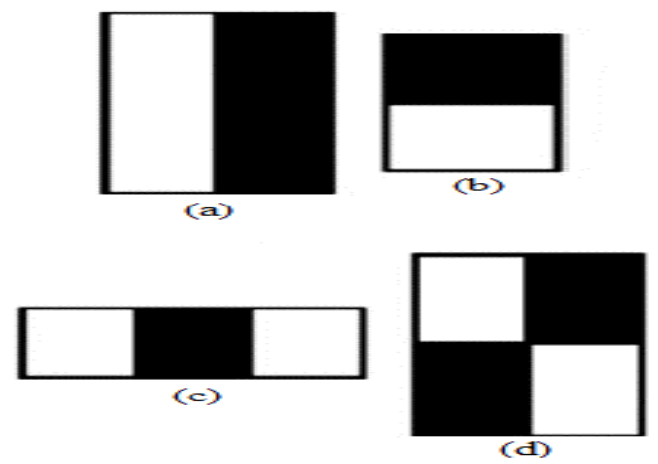

Fig 5: Haar Features

\subsection{Yawning Detection}

Yawning detection is done to verify the validity of the detected component. When mouth start to open then threshold pixel value is also increases as compared to normal position of mouth which is nothing but yawning state following are the steps

1. Get eye highlighted region.

2. Get mouth highlighted region.

3. Get yawn mouth pixel count YI and normal mouth pixel count NI.

4. Get yawn eye pixel count YE and normal eye pixel count NE.

5. If $(\mathrm{YI}>\mathrm{NI})$ or $(\mathrm{YE}<\mathrm{NE})$ then

6. Driver is yawning.

\section{CONCLUSIONS}

In this paper, we have presented driver drowsiness detection method based on yawning detection. The face detection system presented deals with detection of skin like color regions in the YCbCr. Our aim is to prevent road accidents. In future, the proposed system can modified for the live cameras in vehicles.

\section{ACKNOWLEDGEMENTS}

The author wish to acknowledge Pune university of Maharashtra for providing grateful support and necessary help provided for the work.

\section{REFERENCES}

[1] NHTSA, "Drowsy drivers detection and Warning system for commercial vehicle drivers: Field proportional test design, analysis, and progress" National Highway Traffic Safety Administration, Washington, DC.

[2] Ijaz Khan, Hadi Abdullah and Mohd Shamian Bin Zainal. "Efficien Eyes and Mouth Detection Algorithm Combination of Viola Jones and Skin Color Pixel Detection" International Journal of Engineering and Applied Sciences, June 2013. Vol. 3, No. 4.

[3] Varsha Powar, Aditi Jahagirdar, Sumedha Sirsikar "Skin Detection in YCbCr Color Space" International Journal of Computer Applications (0975 - 8887) Volume *-No.*, 2011.

[4] Jaeik Jo Sung Joo Lee, Ho Gi Jung, Kang Ryoung Park,Jaihie Kim "Vision-based method for detecting driver drowsiness and distraction in driver monitoring system " Optical Engineering 50(12), 127202 (December 2011)

[5] Monali V. Rajput,J. W. Bakal " Execution Scheme for Driver Drowsiness Detection using Yawning Feature" International Journal of Computer Applications (0975 8887) Volume 62- No.6, January 2013

[6] Hui-Yu Huang and Yan-Ching Lin "An Efficient Mouth Detection Based on Face Localization and Edge Projection" International Journal of Computer Theory and Engineering, Vol. 5, No. 3, June 2013

[7] Swapnil V Tathe and Sandipan P Narote "Face detection using color models" World Journal of Science and Technology 2012, ISSN: $2231-2587$

[8] Garima Turan, Sheifali Gupta "Road Accidents Prevention system using Driver's Drowsiness Detection" International Journal of Advanced Research in Computer Engineering \& Technology (IJARCET) Volume 2, Issue 11, November 2013

[9] Shabnam Abtahi, Behnoosh Hariri, Shervinshir mohammadi,"Driver Drowsiness Monitoring based on Yawning Detection" Distributed Collaborative Virture Environment Research Laboratory University of Ottawa, Ottawa, Canada, 2011. 
[10] Ms.Prajakta M.Patil Prof.Y.M.Patil "Robust Skin Colour Detection And Tracking Algorithm" International Journal of Engineering Research \& Technology (IJERT)Vol. 1 Issue 8, October - 2012 ISSN: 2278-0181

\section{BIOGRAPHIES}

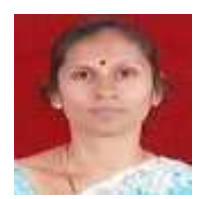

Prof.G.M.Bhandari,M.E(Computer),HOD of computer dept. JSPM's Bhivarabai Sawant Institute Of Technology and Research(Women),Wagholi,Pune-412207

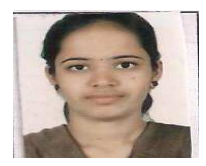

Archana Durge, Computer Department, JSPM's Bhivarabai Sawant Institute Of Technology and Research (Women), Wagholi ,Pune-412207

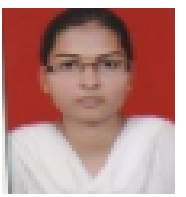

Aparna Bidwai, Computer Department, JSPM's Bhivarabai Sawant Institute Of Technology and Research (Women), Wagholi ,Pune-412207

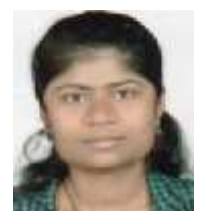

Urmila Aware, Computer Department, JSPM's Bhivarabai Sawant Institute Of Technology and Research (Women), Wagholi , Pune-412207 